\title{
The effective number of neutrinos: standard and non-standard scenarios
}

\author{
Sergio Pastor ${ }^{* \dagger}$ \\ Institut de Física Corpuscular (CSIC-Universitat de València) \\ Parc Científic UV, C/ Catedrático José Beltrán, 2 \\ E-46980 Paterna (Valencia), Spain \\ E-mail: pastor@ific.uv.es
}

\begin{abstract}
We study the decoupling process of neutrinos in the early universe in the presence of threeflavour oscillations. The evolution of the neutrino spectra is found by solving the corresponding momentum-dependent kinetic equations for the neutrino density matrix, including for the first time the proper collision integrals for both diagonal and off-diagonal elements. We find that the contribution of neutrinos to the cosmological energy density in the form of radiation, in terms of the effective number of neutrinos, is $N_{\text {eff }}=3.045$. This result does not depend on the ordering of neutrino masses, it is in agreement with previous theoretical calculations and consistent with the latest analysis of Planck data. We also calculate the effect of non-standard neutrinoelectron interactions (NSI), predicted in many theoretical models where neutrinos acquire mass. For two sets of NSI parameters allowed by present data, we find that $N_{\text {eff }}$ can be reduced down to 3.040 or enhanced up to 3.059 . Finally, we consider the case of very low reheating scenarios $\left(T_{\mathrm{RH}} \sim \mathscr{O}(\mathrm{MeV})\right)$, where the thermalization of neutrinos can be incomplete $\left(N_{\mathrm{eff}}<3\right)$ and leads to a lower bound on the reheating temperature, $T_{\mathrm{RH}}>4.7 \mathrm{MeV}$ from Planck data (95\% CL).
\end{abstract}

Neutrino Oscillation Workshop

4 - 11 September, 2016

Otranto (Lecce, Italy)

\footnotetext{
* Speaker.

${ }^{\dagger}$ Work supported by the Spanish grants FPA2014-58183-P, Multidark CSD2009-00064 and SEV-2014-0398 (MINECO) and PROMETEOII/2014/084 (Generalitat Valenciana).
} 


\section{Introduction}

The existence of a relic background of neutrinos, produced in the early universe, is a generic prediction of the hot big bang model. These elusive particles were in thermal equilibrium with charged leptons and the rest of the primeval plasma, until the cosmic temperature dropped below $2-3 \mathrm{MeV}$ and neutrinos decoupled. In the simple but accurate approximation known as instantaneous decoupling, neutrinos kept the energy distribution of a relativistic fermion, unchanged but for the effect of redshift of physical momentum, and did not share the entropy release from electronpositron annihilations into photons. This leads to to the well-known ratio of temperatures of relic photons and neutrinos, $T_{\gamma} / T_{V}=(11 / 4)^{1 / 3}$, and to a contribution of neutrinos to the cosmological energy density of radiation given, in terms of the effective number of neutrinos, by $N_{\text {eff }}=3$ [1].

Neutrino decoupling and $e^{ \pm}$annihilations are so close in time that some relic interactions exist below the decoupling temperature. These processes, more efficient for neutrinos with larger momenta, lead to non-thermal distortions in the neutrino spectra at the percent level and a slightly smaller increase of the comoving photon temperature, as calculated in a series of papers (see the more recent works $[2,3,4]$ and references therein). The value found for $N_{\text {eff }}$ is only $0.04-$ 0.05 larger than the number in the instantaneous decoupling approximation, but it is an important parameter because it fixes the contribution of neutrinos in the standard case.

Prompted by the increased accuracy on the current measurements of $N_{\text {eff }}$ (for instance, the last analysis with Planck data found $N_{\text {eff }}=3.15 \pm 0.23$ at 68\% CL [5]), we have revisited the process of neutrino decoupling in the early universe with the aim of checking some of the approximations assumed in previous works. We have solved the Boltzmann equations for the neutrino density matrix with full collision integrals, for both the diagonal and off-diagonal terms, avoiding for the latter the so-called damping factors previously used. We also consider three-flavour neutrino oscillations (with present best-fit values of the mixing parameters) and investigate whether the results depend on the two possible ways of ordering the neutrino masses: normal (NH) or inverted (IH) hierarchy. In addition, we consider two non-standard scenarios that can modify neutrino decoupling and the final value of $N_{\text {eff }}$. First, we update the analysis in [6] and include the presence of non-standard neutrino-electron interactions allowed by laboratory data. Finally, we consider a very low reheating scenario, where the final cosmological period dominated by relativistic particles in thermal equilibrium starts at a maximum temperature $T_{\mathrm{RH}}$ of the order of a few $\mathrm{MeV}$. In such a case, the thermalization of neutrinos could be incomplete due to the lack of interactions and $N_{\text {eff }}<3$.

\section{Results in the standard case}

First, we consider the case with standard weak interactions. For all technical details about the numerical calculations of the evolution of the neutrino distribution functions, we refer the reader to [7]. We use the frozen values of the neutrino spectra, once weak interactions are completely ineffective, to calculate how the energy density of each flavour state is enhanced with respect to the instantaneous decoupling approximation. In all cases the enhancement is at the sub-percent level, which indicates the well-known result that the non-thermal distortions for cosmological neutrinos are very small. The main effect is a slightly larger contribution of neutrinos to the cosmological energy density of radiation $\rho_{\mathrm{r}}$, usually given, as in the case of any other contribution of relativistic 


\begin{tabular}{|l|ccccc|}
\hline Case & $z_{\text {fin }}$ & $\delta \bar{\rho}_{v_{e}}(\%)$ & $\delta \bar{\rho}_{v_{\mu}}(\%)$ & $\delta \bar{\rho}_{v_{\tau}}(\%)$ & $N_{\text {eff }}$ \\
\hline Instantaneous decoupling & 1.40102 & 0 & 0 & 0 & 3.000 \\
Inst. dec. (with QED corrections) & 1.39975 & 0 & 0 & 0 & 3.011 \\
No oscillations & 1.39784 & 0.920 & 0.392 & 0.392 & 3.045 \\
\hline Normal hierarchy (NH) & 1.39779 & 0.699 & 0.511 & 0.519 & 3.045 \\
NH (damping terms) & 1.39778 & 0.673 & 0.529 & 0.533 & 3.045 \\
Inverted hierarchy (IH) & 1.39779 & 0.697 & 0.512 & 0.520 & 3.045 \\
\hline NSI $e e(\mathrm{NH})$ & 1.39812 & 0.592 & 0.460 & 0.467 & 3.040 \\
NSI $\tau \tau(\mathrm{NH})$ & 1.39708 & 0.862 & 0.784 & 0.812 & 3.059 \\
\hline
\end{tabular}

Table 1: Final values of the dimensionless photon temperature, the distortion of the energy densities of flavour neutrinos and the effective number of neutrinos, computed for several cases as discussed in the text.

particles other than photons, in terms of the effective number of neutrinos,

$$
N_{\text {eff }}=\frac{8}{7}\left(\frac{11}{4}\right)^{4 / 3}\left[\frac{\rho_{\mathrm{r}}-\rho_{\gamma}}{\rho_{\gamma}}\right]=\left(\frac{z_{0}}{z_{\text {fin }}}\right)^{4}\left(3+\delta \bar{\rho}_{v_{e}}+\delta \bar{\rho}_{v_{\mu}}+\delta \bar{\rho}_{v_{\tau}}\right), \text { where } \delta \bar{\rho}_{v_{\alpha}} \equiv \frac{\rho_{v_{\alpha}}-\rho_{v_{0}}}{\rho_{v_{0}}} .
$$

Here $z_{0}=(11 / 4)^{1 / 3}$ and $z_{\text {fin }}$ are the asymptotic values of the dimensionless photon temperature in the instantaneous decoupling approximation and for each case in our calculations, respectively, and $\rho_{v_{0}}$ is the energy density of one neutrino state that does not share the entropy release from the $e^{ \pm}$ annihilations. The final values of $z_{\text {fin }}, \delta \bar{\rho}_{v_{\alpha}}$ and $N_{\text {eff }}$ for all cases are given in Table 1.

Our main results correspond to the cases $\mathrm{NH}$ and $\mathrm{IH}$. Computed with the full collision terms, both lead to almost equal values of the enhanced neutrino energy densities and $z_{\text {fin }}$, and to the same $N_{\text {eff }}=3.045$. We conclude that the value of $N_{\text {eff }}$ does not depend on the ordering of neutrino masses. Fixing it to $\mathrm{NH}$, we find that, within the accuracy of our numerical calculations, $N_{\text {eff }}$ is the same when the off-diagonal collision terms are calculated with the full integrals or with the simple damping prescription. There exist small differences in $\delta \bar{\rho}_{v_{\alpha}}$, that are slightly smaller (larger) for muon or tau (electron) neutrinos when the damping approximation is not used. However, for practical purposes, in particular concerning $N_{\text {eff }}$, using the full collision integrals in the evolution equation of the off-diagonal components is not necessary. Our results for the case of zero mixing, also shown in Table 1 (again with $N_{\text {eff }}=3.045$ ), can be compared with those found in previous works for zero neutrino mixing including QED corrections: 3.046 [2], 3.044 [3] and 3.052 [4].

\section{Non-standard scenarios}

Most extended particle physics models where neutrinos acquire mass also lead to new nonstandard interactions (NSI) involving neutrinos. Here we show the effects of NSI on relic neutrino decoupling with a couple of examples, fixing the mass hierarchy to $\mathrm{NH}$. We consider two sets of NSI parameters (only neutrino-electron interactions are relevant for our problem), where either $\varepsilon_{e e}^{P}$ or $\varepsilon_{\tau \tau}^{P}$ are nonzero [7]. Our results are listed in the lower rows of Table 1, where one can see that the presence of NSI modifies the neutrino spectral distortions and leads to small changes in the final value of $N_{\text {eff }}$ : positive (0.014) for the $\tau \tau$ case and negative $(-0.005)$ when $\varepsilon_{e e} \neq 0$.

We have also studied the relic neutrino decoupling in a generic cosmological model where the latest reheating phase occurs at temperatures as low as $1 \mathrm{MeV}$. We have carefully solved the 


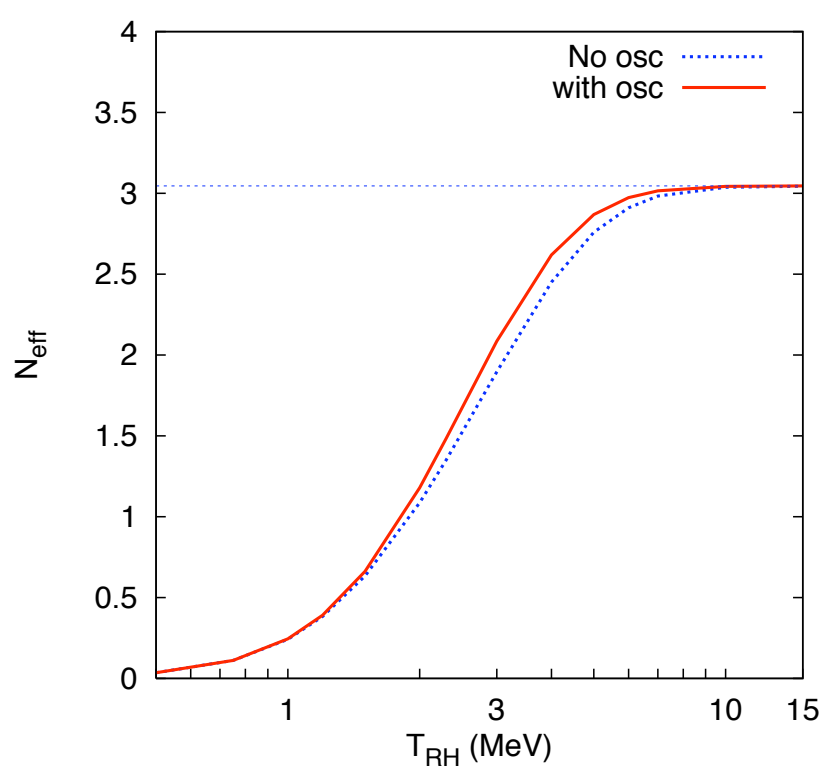

Figure 1: Final contribution of neutrinos to the radiation energy density in terms of $N_{\text {eff }}$, as a function of the reheating temperature. The horizontal line indicates the standard value, $N_{\text {eff }}=3.045$.

thermalization of neutrinos when $T_{\mathrm{RH}}<15 \mathrm{MeV}$, improving previous calculations and taking into account three-flavour neutrino oscillations. As shown in figure 1, the final $N_{\text {eff }}$ can be smaller than the standard value. A stringent bound on the reheating temperature can be obtained from the analysis of CMB anisotropies. With the same basic dataset used by the Planck collaboration, we find the lower limit $T_{\mathrm{RH}} \geq 4.7 \mathrm{MeV}$ (95\% CL), if neutrino masses are allowed to vary.

\section{References}

[1] J. Lesgourgues, G. Mangano, G. Miele and S. Pastor, Neutrino cosmology, Cambridge Un. Press 2013.

[2] G. Mangano et al, Relic neutrino decoupling including flavour oscillations, Nucl. Phys. B 729 (2005) 221-234 [hep-ph/0506164].

[3] J. Birrell, C.T. Yang and J. Rafelski, Relic Neutrino Freeze-out: Dependence on Natural Constants, Nucl. Phys. B 890 (2014) 481-517 [arXiv:1406.1759].

[4] E. Grohs, G.M. Fuller, C.T. Kishimoto, M.W. Paris and A. Vlasenko, Neutrino energy transport in weak decoupling and big bang nucleosynthesis, Phys. Rev. D 93 (2016) 083522 [arXiv:1512.02205].

[5] P.A.R. Ade et al [Planck Collaboration], Planck 2015 results. XIII. Cosmological parameters, Astron. Astrophys. 594 (2016) A13 [arXiv:1502.01589].

[6] G. Mangano et al, Effects of non-standard neutrino-electron interactions on relic neutrino decoupling, Nucl. Phys. B 756 (2006) 100-116 [hep-ph/0607267].

[7] P.F. de Salas and S. Pastor, Relic neutrino decoupling with flavour oscillations revisited, JCAP 07 (2016) 051 [arXiv:1606.06986].

[8] P.F. de Salas, M. Lattanzi, G. Mangano, G. Miele, S. Pastor and O. Pisanti, Bounds on very low reheating scenarios after Planck, Phys. Rev. D 92 (2015) 123534 [arXiv:1511.00672]. 